\title{
Feasibility of $\alpha$-fetoprotein as a diagnostic tool for hepatocellular carcinoma in Korea
}

\author{
Dae Geon Ahn ${ }^{1}$, Hyung Joon Kim ${ }^{1}$, Hyun Kang ${ }^{2}$, Hyun Woong Lee ${ }^{1}$, Si Hyun Bae , Joon Hyoek Lee , \\ Yong Han Paik ${ }^{4}$, and June Sung Lee
}

Departments of ${ }^{1}$ Internal Medicine and ${ }^{2}$ Anesthesiology and Pain, Chung-Ang University College of Medicine, Seoul; ${ }^{3}$ Department of Internal Medicine, College of Medicine, The Catholic University of Korea, Seoul; ${ }^{4}$ Department of Medicine, Samsung Medical Center, Sungkyunkwan University School of Medicine, Seoul; ' Department of Internal Medicine, Inje University Ilsan Paik Hospital, Goyang, Korea

Received: April 1, 2014

Revised : October 4, 2014

Accepted: October 17, 2014

\section{Correspondence to}

Hyung Joon Kim, M.D.

Department of Internal Medicine, Chung-Ang University College of Medicine, 84 Heukseok-ro, Dongjak-gu, Seoul o6974, Korea

Tel: $+82-2-6299-1376$

Fax: +82-2-825-7571

E-mail: mdjoon@cau.ac.kr
Background/Aims: The aim of this study was to evaluate the feasibility of $\alpha$-fetoprotein (AFP) as a diagnostic tool for hepatocellular carcinoma (HCC) in Korean patients.

Methods: We retrospectively reviewed the medical records of HCC and cirrhosis patients at three hospitals. For each HCC patient, a cirrhosis patient matched for age, sex, etiology, and Child-Pugh classification was selected by simple random sampling. The performance of AFP in the diagnosis of HCC was determined using receiver operating characteristic curve analysis.

Results: A total of 732 patients with HCC or cirrhosis were selected for each case and the control groups. The mean age was 54 years, and $72.4 \%$ of patients were male. The mean serum AFP levels in the HCC group and cirrhosis group were $3,315.6$ and $117.2 \mathrm{ng} / \mathrm{mL}$, respectively $(p<0.001)$. The area under the receiver operating characteristic curve for all HCC patients was 0.757 . The sensitivity, specificity, and positive predictive value of AFP was 50.55\%, 87.70\%, and 80.43\%, respectively, at a cut-off of $20 \mathrm{ng} / \mathrm{mL} ; 37.70 \%, 95.90 \%$, and $90.20 \%$, respectively, at a cutoff of $100 \mathrm{ng} / \mathrm{mL}$, and $30.05 \%, 97.27 \%$, and $91.67 \%$, respectively, at a cut-off of 200 $\mathrm{ng} / \mathrm{mL}$. A cut-off of $100 \mathrm{ng} / \mathrm{mL}$ was more sensitive than one of $200 \mathrm{ng} / \mathrm{mL}$ with equivalent specificity and positive predictive value.

Conclusions: The cut-off AFP value for early-stage HCC was $17.4 \mathrm{ng} / \mathrm{mL}$. Our study cautiously suggests that AFP has a role in the diagnosis of HCC, and that the appropriate value of AFP for the diagnosis of HCC may be $100 \mathrm{ng} / \mathrm{mL}$ rather than $200 \mathrm{ng} / \mathrm{mL}$.

Keywords: Alpha-fetoproteins; Carcinoma; Diagnosis; Hepatocellular

\section{INTRODUCTION}

Hepatocellular carcinoma (HCC) is the most common primary malignancy of the liver and the third most common cause of cancer-related death worldwide [1]. Whereas effective treatment modalities exist to cure early-stage HCC, including surgical resection, radiofrequency ablation, and alcohol injection, late-stage HCC has few therapeutic options [2]. Furthermore, difficulty in early detection of HCC is related to poor prognosis, and more than two-thirds of patients are diagnosed at advanced stages [3].

In 1963, an antigen specific to human HCC was first reported by Yuri S. Tatarinov, and in 1970, it was given the name " $\alpha$-fetoprotein" (AFP) [4]. This antigen has long been recognized as the first oncodevelopmental biomarker and has been used as a screening and diagnostic tool for HCC [4-6]. However, with the widespread 
use of advanced imaging techniques, the usefulness of the AFP assay in the diagnosis of HCC has decreased $[7,8]$. The sensitivity and specificity of AFP vary widely based on study design and patient characteristics [7,911]; recent guidelines do not recommend the use of AFP as a diagnostic test $[8,12]$, focusing instead on the typical contrast enhancement imaging patterns of arterial hypervascularity with washout in the portal venous or delayed phases.

However, some debate still exists about the utility of AFP in the early diagnosis of HCC [13], and the role of AFP among different etiologic and ethnic groups remains to be established [14,15]. Moreover, other studies still highlight the diagnostic value of AFP $[13,16]$.

As no well-designed studies in Koreans are available, the usefulness of AFP for HCC diagnosis in the Korean population remains unclear. We hypothesized that AFP may still have a role as a diagnostic test for HCC in the Korean population. Thus, we aimed to evaluate the feasibility of using AFP as a diagnostic tool for HCC and to determine the best cut-off value of serum AFP for discriminating HCC and cirrhosis.

\section{METHODS}

\section{Patients}

Between January 2005 and September 2012, we identified consecutive cases of newly diagnosed HCC at three university-affiliated hospitals (the Samsung Medical Center, Seoul St. Mary's Hospital, and Chung-Ang University Hospital) in Seoul, Republic of Korea. For each HCC patient, we selected a cirrhosis control patient matched for age, sex, etiology, and Child-Pugh classification (CPC) by simple random sampling. The ages were stratified by decade. Controls were selected during the same time period as the cases at these centers. The following available data were collected from all cases: past medical history, demographic information, underlying etiologies of HCC or cirrhosis, serum AFP, CPC, severity and complication of liver cirrhosis, radiologic findings, and performance status at the time of diagnosis.

\section{Diagnosis and definitions}

We established the diagnosis of HCC by histological examination, and staging was determined using the
Barcelona Clinic Liver Cancer (BCLC) staging system [5]. BCLC stage A (early HCC) is defined by a single lesion between 2 and $5 \mathrm{~cm}$ in size, or by $\leq 3$ lesions each $<3 \mathrm{~cm}$ in size, without portal vein thrombosis or extrahepatic metastasis. BCLC stage o (very early HCC) is defined as a single lesion $<2 \mathrm{~cm}$ without vascular involvement or metastasis. "Early-stage" HCC included BCLC stage A and BCLC stage o. "Late-stage" HCC is defined as the combination of intermediate (BCLC stage B) and advanced (BCLC stage C) HCC [5]. Those with end-stage or significant medical comorbidities, in which survival was predicted to be less than 1 year, were excluded.

The presence of cirrhosis was defined by histology or by evidence of unequivocal clinical and laboratory evidence of cirrhosis, such as ultrasound (US) and/or computed tomography (CT) findings indicating cirrhosis (an irregular liver surface, splenomegaly, etc.) and the detection of signs/symptoms consistent with decompensated cirrhosis (jaundice, varices due to portal hypertension, ascites, or hepatic coma) [17].

Ascites was diagnosed by abdominal CT or US. The underlying etiology of HCC was related to hepatitis B virus (HBV) infection if serological detection of the hepatitis B surface antigen was positive. Hepatitis $\mathrm{C}$ virus (HCV) infection was diagnosed if patients were seropositive for the antibody against HCV (anti-HCV) by a second-generation enzyme immunoassay. Alcoholism was diagnosed in subjects with a documented history of alcohol excess of at least $40 \mathrm{~g}$ of alcohol daily for 5 years or more. The etiology of liver disease was based on the judgment of the attending physician.

The Child-Pugh classification was evaluated based on serum levels of albumin and bilirubin, prothrombin time prolongation, and the severity of ascites and encephalopathy. All patients were treatment-naïve, and none had received specific anticancer treatments at the time of diagnosis.

\section{Statistical methods}

Statistical significance was assessed using the chi-square test or the Fisher exact test for categorical variables and the Student $t$ test or the Mann-Whitney Utest for continuous variables. Our study was designed to compare the optimal cut-off values of AFP by maximizing the sum of sensitivity and specificity. For the analysis of the accuracy of AFP, a receiver operating characteristic (ROC) curve 
was plotted for AFP. The area under the curve (AUC) for the ROC curve (AUROC) of each group was calculated. For all comparisons, a $p<0.05$ was considered statistically significant, and all analyses were performed using SAS version 9.13 (SAS, Cary, NC, USA).

\section{Ethics statement}

This study conformed to the standards of the Declaration of Helsinki and current ethical guidelines and was approved by the Institutional Review Board at ChungAng University Hospital (C2012174[869]).

\section{RESULTS}

\section{Patients}

A total of 732 patients with HCC and cirrhosis were selected for each of the case and control groups. Among all HCC patients, 268 were classified with early-stage HCC ( $n=36$ very early, $n=232$ early) and 98 were classi- fied as having late-stage HCC. Baseline characteristics of these patients are shown in Table 1. The mean age was 55.5 years, and $72.4 \%$ of the patients were male. The most common etiology of chronic liver disease was HBV (65.9\%), followed by HCV (12.8\%), and alcoholism (13.1\%).

\section{AFP level}

The mean and median serum AFP levels in the HCC and cirrhosis groups are shown in Table 2. The mean serum AFP levels in the HCC and cirrhosis groups were 3,315.6 and $117.2 \mathrm{ng} / \mathrm{mL}$, respectively $(p<0.001)$. In the HCC group, late-stage HCC patients showed a significantly higher mean AFP level than early-stage HCC patients (7,994.7 ng/mL vs. 1,604.6 ng/mL, $p<0.001)$.

\section{AUC of AFP}

When all patients with HCC were evaluated, the AUROC for total AFP was 0.757 , and the AUROC of early-stage and late-stage HCC were 0.737 and 0.807 , respectively (Fig. 1). When maximizing the sum of sensitivity and

\section{Table 1. Baseline patient characteristics}

\begin{tabular}{|c|c|c|c|}
\hline Characteristic & HCC cases $(n=366)$ & Cirrhosis controls $(n=366)$ & Total $(\mathrm{n}=732)$ \\
\hline Age, yr, & $55.64 \pm 10.93$ & $55.39 \pm 11.23$ & $55.52 \pm 11.08$ \\
\hline \multicolumn{4}{|l|}{ Sex } \\
\hline Male & $265(72.40)$ & $265(72.40)$ & $530(72.40)$ \\
\hline Female & $101(27.60)$ & $101(27.60)$ & $202(27.60)$ \\
\hline \multicolumn{4}{|l|}{ Etiology } \\
\hline Alcohol & $48(13.11)$ & $48(13.11)$ & $96(13.11)$ \\
\hline HBV & $241(65.85)$ & $241(65.85)$ & $482(65.85)$ \\
\hline $\mathrm{HCV}$ & $47(12.84)$ & $47(12.84)$ & $94(12.84)$ \\
\hline $\mathrm{NBNC}$ & $30(8.20)$ & $30(8.20)$ & $60(8.20)$ \\
\hline \multicolumn{4}{|c|}{ Child-Pugh class } \\
\hline A & $302(82.51)$ & $302(82.51)$ & $604(82.51)$ \\
\hline $\mathrm{B}$ & $54(14 \cdot 75)$ & $54(14 \cdot 75)$ & $108(14.75)$ \\
\hline $\mathrm{C}$ & $10(2.73)$ & $10(2.73)$ & $20(2.73)$ \\
\hline \multicolumn{4}{|l|}{ BCLC stage } \\
\hline 0 & $36(9.84)$ & & $36(9.84)$ \\
\hline A & $232(63.39)$ & & $232(63.39)$ \\
\hline $\mathrm{B}$ & $35(9.56)$ & & $35(9.56)$ \\
\hline $\mathrm{C}$ & $63(17.21)$ & & $63(17.21)$ \\
\hline
\end{tabular}

Values are presented as mean \pm SD or number (\%).

HCC, hepatocellular carcinoma; HBV, hepatitis B virus; HCV, hepatitis C virus; NBNC, non-B non-C; BCLC, Barcelona Clinic Liver Cancer. 
Table 2. Mean and median values of AFP in the HCC and cirrhosis groups

\begin{tabular}{|c|c|c|c|c|c|}
\hline \multirow{2}{*}{ Variable } & \multicolumn{3}{|c|}{ HCC group } & \multirow{2}{*}{ Cirrhosis group } & \multirow{2}{*}{ Overall } \\
\hline & Early stage & Late stage & All & & \\
\hline Number & 268 & 98 & 366 & 366 & 732 \\
\hline \multicolumn{6}{|c|}{ AFP, ng/mL } \\
\hline Mean & $1,604.6$ & $7,994 \cdot 7$ & $3,315.6$ & 117.2 & $1,716.4$ \\
\hline $\mathrm{SD}$ & $7,730.1$ & 25,309 & 14,899 & $1,787.4$ & 10,723 \\
\hline Median & 19.05 & 34.07 & 20.45 & 3.95 & 6.86 \\
\hline Min & 1.00 & 1.02 & 1.00 & 0.57 & 0.57 \\
\hline Max & 10,221 & 200,000 & 200,000 & 34,150 & 200,000 \\
\hline
\end{tabular}

AFP, $\alpha$-fetoprotein; HCC, hepatocellular carcinoma; SD, standard deviation.

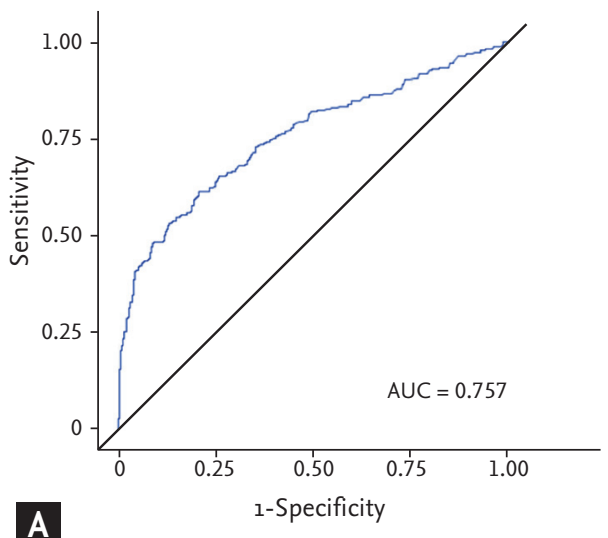

Figure 1. Receiver operating characteristic (ROC) curves showing the sensitivity and specificity of serum $\alpha$-fetoprotein values in the diagnosis of hepatocellular carcinoma (HCC) in (A) all HCC cases ( $n=366$ ), (B) early-stage HCC cases ( $\mathrm{n}=268$ ), and (C) late-stage HCC cases $(n=98)$. AUC, area under the curve.

specificity for the AUROC curves, the optimal value of AFP was $10.8 \mathrm{ng} / \mathrm{mL}$ in all HCC groups (sensitivity $61.48 \%, 95 \%$ confidence interval [CI], 56.49 to 66.46 ; specificity $79.23 \%, 95 \% \mathrm{CI}, 75.08$ to 83.39 ), $17.4 \mathrm{ng} / \mathrm{mL}$ in the early-stage HCC group (sensitivity 52.24\%, 95\% CI, 46.26 to 58.22 ; specificity $86.89 \%, 95 \%$ CI, 83.43 to 90.34 ), and $11.0 \mathrm{ng} / \mathrm{mL}$ (sensitivity $66.33 \%, 95 \% \mathrm{CI}, 56.97$ to 75.68 ; specificity $79.23 \%, 95 \% \mathrm{CI}, 75.08$ to 83.39 ) in the late-stage HCC group (Table 3). In the early-stage HCC group, the optimal value of AFP was higher for amviral etiology than nonviral etiologies $(18.0 \mathrm{ng} / \mathrm{mL}$ vs. $5.1 \mathrm{ng} / \mathrm{mL}, p<$ 0.001).

\section{Sensitivity, specificity, and positive predictive value of AFP}

The sensitivity, specificity, and positive predictive val- ue (PPV) for the various AFP cut-off values in each HCC group are presented in Table 4. In all patients, a cut-off value of $20 \mathrm{ng} / \mathrm{mL}$ showed a sensitivity of 50.55\% (95\% CI, 45.42 to 55.67 ), a specificity of $87.70 \%$ ( $95 \%$ CI, 84.34 to 91.07), and a PPV of 80.43\% (95\% CI, 75.31 to 85.56). Moreover, a cut-off value of $100 \mathrm{ng} / \mathrm{mL}$ showed a sensitivity of $37.70 \%$ (95\% CI, 32.74 to 42.67 ), a specificity of $95.90 \%$ (95\% CI, 93.87 to 97.93), and a PPV of $90.20 \%$ (95\% CI, 85.47 to 94.91 ), while a cut-off value of $200 \mathrm{ng} / \mathrm{mL}$ showed a sensitivity of $30.05 \%$ (95\% CI, 25.36 to 34.75$)$, a specificity of $97.27 \%$ (95\% CI, 95.60 to 98.94), and a PPV of $91.67 \%$ (95\% CI, 86.72 to 96.61). In the early-stage, late-stage, and overall HCC groups, a cut-off value of $100 \mathrm{ng} / \mathrm{mL}$ showed higher sensitivity than one of $200 \mathrm{ng} / \mathrm{mL}$, without compromising specificity or PPV. 
Table 3. Cut-off values of AFP at the maximal sensitivity and specificity in the receiver operating characteristics

\begin{tabular}{lccc}
\hline \multirow{2}{*}{ Variable } & \multicolumn{3}{c}{ AFP, $\mathrm{ng} / \mathrm{mL}$} \\
\cline { 2 - 4 } & Cut-off & Sensitivity, \% (95\% CI) & Specificity, \% (95\% CI) \\
\hline All HCC $(\mathrm{n}=366)$ & 10.8 & $61.48(56.49-66.46)$ & $79.23(75.08-83.39)$ \\
Early-stage HCC $(\mathrm{n}=268)$ & 17.4 & $52.24(46.26-58.22)$ & $86.89(83.43-90.34)$ \\
Late-stage HCC $(\mathrm{n}=98)$ & 11.0 & $66.33(56.97-75.68)$ & $79.23(75.08-83.39)$ \\
Viral etiology and early stage $(\mathrm{n}=216)$ & 18.0 & $56.48(49.87-63.09)$ & $86.89(83.43-90.34)$ \\
Non-viral etiology and early stage $(\mathrm{n}=52)$ & 5.1 & $67.31(54.56-80.06)$ & $61.20(56.21-66.19)$ \\
\hline
\end{tabular}

AFP, $\alpha$-fetoprotein; CI, confidence interval; HCC, hepatocellular carcinoma.

Table 4. Sensitivity, specificity, and PPV of AFP in early-stage, late-stage, and all HCC groups

\begin{tabular}{|c|c|c|c|c|}
\hline \multirow{2}{*}{ Group } & \multicolumn{4}{|c|}{ Marker AFP, ng/mL } \\
\hline & Cut-off & Sensitivity, \% (95\% CI) & Specificity, \% (95\% CI) & PPV, \% (95\% CI) \\
\hline \multirow[t]{6}{*}{ All HCC $(n=366)$} & 5 & $75.14(70.71-79.56)$ & $60.38(55.37-65.39)$ & $65.48(60.93-70.02)$ \\
\hline & 10 & $61.48(56.49-66.46)$ & $76.78(72.45-81.10)$ & $72.58(67.61-77.55)$ \\
\hline & 20 & $50.55(45.42-55.67)$ & $87.70(84.34-91.07)$ & $80.43(75.31-85.56)$ \\
\hline & 100 & $37.70(32.74-42.67)$ & $95.90(93.87-97.93)$ & $90.20(85.48-94.91)$ \\
\hline & 200 & $30.05(25.36-34.75)$ & $97.27(95.60-98.94)$ & $91.67(86.72-96.61)$ \\
\hline & 400 & $24.04(19.67-28.42)$ & $98.36(97.06-99.66)$ & $93.62(88.68-98.56)$ \\
\hline \multirow[t]{6}{*}{ Early-stage HCC $(n=268)$} & 5 & $72.76(67.43-78.09)$ & $60.38(55.37-65.39)$ & $57 \cdot 35(52.10-62.61)$ \\
\hline & 10 & $59.70(53.83-65.57)$ & $76.78(72.45-81.10)$ & $65.31(59 \cdot 35-71.27)$ \\
\hline & 20 & $49.25(43.27-55.24)$ & $87.70(84.34-91.07)$ & $74.58(68.16-80.99)$ \\
\hline & 100 & $34.70(29.00-40.40)$ & $95.90(93.87-97.93)$ & $86.11(79.59-92.63)$ \\
\hline & 200 & $27.24(21.91-32.57)$ & $97.27(95.60-98.94)$ & $87.95(80.95-94.95)$ \\
\hline & 400 & $20.52(15.69-25.36)$ & $98.36(97.06-99.66)$ & $90.16(82.69-97.64)$ \\
\hline \multirow[t]{6}{*}{ Late-stage $\mathrm{HCC}(\mathrm{n}=98)$} & 5 & $81.63(73.97-89.30)$ & $60.38(55.37-65.39)$ & $35.56(29 \cdot 30-41.81)$ \\
\hline & 10 & $66.33(56.97-75.68)$ & $76.78(72.45-81.10)$ & $43.33(35.40-51.26)$ \\
\hline & 20 & $54.08(44.22-63.95)$ & $87.70(84.34-91.07)$ & $54.08(44.22-63.95)$ \\
\hline & 100 & $45.92(36.05-55.78)$ & $95.90(93.87-97.93)$ & $75.00(64.04-85.96)$ \\
\hline & 200 & $37.76(28.16-47.35)$ & $97.27(95.60-98.94)$ & $78.72(67.02-90.42)$ \\
\hline & 400 & $33.67(24.32-43.03)$ & $98.36(97.06-99.66)$ & $84.62(73.29-95.94)$ \\
\hline
\end{tabular}

PPV, positive predictive value; AFP, $\alpha$-fetoprotein; HCC, hepatocellular carcinoma; CI, confidence interval.

\section{DISCUSSION}

We studied the role of AFP as a diagnostic tool for HCC in a Korean population with different HCC prevalence and risk factors compared to other ethnicities $[18,19]$.

Previous reports have demonstrated a relationship between ethnicity and the level of AFP elevation in HCC patients. In Middle Eastern individuals, $20 \%$ of HCC patients had a normal serum AFP level [14], while 38\% of HCC cases in Caucasian individuals did not show AFP elevation [20]. In HCC-related HCC patients, $43 \%$ of African-American and $18 \%$ of a mixed group of Asians, Hispanics, and Caucasians exhibited a normal AFP level [15]. In our cohort of Korean HCC patients, $28 \%$ had normal serum AFP levels. Biological and environmental factors may influence the differences in AFP levels among diverse ethnic groups.

In our study, the mean and median serum AFP levels 
in the HCC group, 3,315.6 and $20.45 \mathrm{ng} / \mathrm{mL}$, respectively, were significantly higher than those in the cirrhosis group, $117.2 \mathrm{ng} / \mathrm{mL}$ and $3.95 \mathrm{ng} / \mathrm{mL}$, respectively. In a Middle Eastern study, median AFP levels were significantly higher in the HCC group (median, $35.4 \mathrm{ng} / \mathrm{mL}$ [range, 0.5 to 805,795$]$ ]) than the cirrhotic group (median, $6.4 \mathrm{ng} / \mathrm{mL}$ [range, 1 to 1,105]; $p<0.0001$ ) [14]. Moreover, the median AFP levels in each cirrhosis group were within the laboratory upper limit of normal. In the early-stage group, the mean AFP level was higher than that in a previous study [21]. This is partially due to several early-stage HCC patients with very high AFP levels; thus, the median AFP value may be more appropriate for comparison.

For the optimal AFP cut-off level of early-stage HCC, nonviral etiology showed higher sensitivity (67.31\% vs. $56.48 \%$ ) but lower specificity (61.20\% vs. $86.89 \%$ ) compared with viral etiology. In one Italian study, a cut-off value of $20 \mathrm{ng} / \mathrm{mL}$ showed a sensitivity of $30.4 \%$ and a specificity of $100.0 \%$ [20]; the sensitivity was lower but the specificity was higher for nonviral etiologies compared with viral etiologies. The majority of nonviral HCC in these two studies were related to alcoholic liver disease, and the difference could be explained in part by ethnicity, environmental factors, and tumor staging. In contrast, a Middle Eastern study in which alcoholic liver disease did not serve as an etiologic factor of nonviral-related HCC, demonstrated that nonviral-related HCC showed the lowest sensitivity (60\%), specificity (29\%), and PPV (16\%) compared with HCV- and HBV-infected groups [14].

The optimal cut-off point of AFP was $10.8 \mathrm{ng} / \mathrm{mL}$ (sensitivity $61.48 \%$ and specificity $79.23 \%$ ) for the diagnosis of all HCC cases and $17.4 \mathrm{ng} / \mathrm{mL}$ (sensitivity $52.24 \%$ and specificity $86.89 \%$ ) for diagnosis of early-stage HCC. Previous studies from the United States and Europe have identified a range of 20 to $30 \mathrm{ng} / \mathrm{mL}$ as the cut-off above which investigations for HCC are needed $[7,15,20]$. However, studies investigating Chinese and Middle Eastern populations found a range from 10 to $12 \mathrm{ng} / \mathrm{mL}$ as the best cut-off $[13,14]$. These latter results are more consistent with those of the current study; the difference may be due to ethnicity, distribution of risk factors, and environmental factors.

In a Chinese study, the optimal cut-off point of AFP for the diagnosis of HCC was $10 \mathrm{ng} / \mathrm{mL}$, with a sensi- tivity of $82.6 \%$ and a specificity of $70.4 \%$, and a level of $200 \mathrm{ng} / \mathrm{mL}$ was suggested to be a relevant cut-off for HCC diagnosis (sensitivity $47.7 \%$, specificity $97.1 \%$, and PPV 97.5\%) [13]. In our study, an AFP value of $100 \mathrm{ng} / \mathrm{mL}$ showed higher sensitivity than one of $200 \mathrm{ng} / \mathrm{mL}$, without compromising specificity or PPV. A cut-off of $20 \mathrm{ng} /$ $\mathrm{mL}$ increased sensitivity at the expense of specificity and PPV, and that of $400 \mathrm{ng} / \mathrm{mL}$ had low sensitivity, limiting its clinical application.

Sanai et al. [14] conducted a multicenter, case-control study involving 206 HCC and 199 cirrhosis cases. The best cut-off point of AFP for the diagnosis of HCC was $11.7 \mathrm{ng} / \mathrm{mL}$, with a sensitivity of $68.4 \%$ and a specificity of 75.4\%. They compared AFP cut-offlevels of 102, 200, and $400 \mathrm{ng} / \mathrm{mL}$ and concluded that an AFP level greater than $100 \mathrm{ng} / \mathrm{mL}$ had a high degree of specificity and could be used as a confirmatory test. The diagnosis of HCC was established on the basis of the published guideline [5] and included the presence of hepatic lesions with typical arterial hypervascularization and washout in the early or delayed venous phase as detected by liver CT and/ or magnetic resonance imaging. They did not use AFP as one of the diagnostic criteria to exclude incorporation bias. However, in clinical practice, it is not uncommon to encounter suspicious malignant cases without typical imaging features. Therefore, in the current study, we included only tissue-confirmed patients to avoid diagnostic uncertainty and false positivity; this feature enabled the assessment of the performance of AFP in the diagnosis of HCC. The results of the two aforementioned studies that targeted Asian populations were similar to ours. This may reflect the distinct secretory function of AFP among racial groups.

The present study has several limitations. First, this was a retrospective study that may have been affected by selection bias. The authors tried to minimize this problem by matching underlying clinical characteristics of case and control patients. As previously reported, HCC patients were older and had a male predominance [14]; we matched age and sex as well as etiology and CPC by simple random sampling. Second, the correlation of AFP levels with contrast enhanced dynamic imaging could not be investigated, as radiologic information was not available in many subjects. However, we diagnosed HCC by histological evidence, which is objective and does not create bias by the attending physician. Third, 
although tumor size is a known independent factor of elevated AFP levels [22], we could not analyze the performance of AFP according to tumor size. Instead, we presented tumor staging, which included rough information about tumor size.

In conclusion, our study showed that the cut-off AFP value for early-stage HCC was $17.4 \mathrm{ng} / \mathrm{mL}$. As a diagnostic tool for HCC, a cut-off value of $100 \mathrm{ng} / \mathrm{mL}$ is more sensitive than one of $200 \mathrm{ng} / \mathrm{mL}$, with similar specificity and PPV. We cautiously suggest that AFP still has a role in the diagnosis of HCC, with an appropriate AFP value of $100 \mathrm{ng} / \mathrm{mL}$ rather than $200 \mathrm{ng} / \mathrm{mL}$. This is the first large multicenter case-control study of Korean HCC patients. However, larger well-designed prospective studies are warranted to validate the optimal AFP value presented in the current study.

\section{KEY MESSAGE}

1. The cut-off $\alpha$-fetoprotein (AFP) value for early-stage hepatocellular carcinoma (HCC) is 17.4 $\mathrm{ng} / \mathrm{mL}$.

2. As a diagnostic tool for HCC, a cut-off of $100 \mathrm{ng} /$ $\mathrm{mL}$ is more sensitive than one of $200 \mathrm{ng} / \mathrm{mL}$, with similar specificity and positive predictive value.

3. AFP still has a role in the diagnosis of HCC, and an appropriate AFP value may be $100 \mathrm{ng} / \mathrm{mL}$ rather than the previously reported value of 200 $\mathrm{ng} / \mathrm{mL}$.

\section{Conflict of interest}

No potential conflict of interest relevant to this article was reported.

\section{REFERENCES}

1. Velazquez RF, Rodriguez M, Navascues CA, et al. Prospective analysis of risk factors for hepatocellular carcinoma in patients with liver cirrhosis. Hepatology 2003;37:520527.

2. Bruix J. Treatment of hepatocellular carcinoma. Hepatology 1997;25:259-262.

3. Collier J, Sherman M. Screening for hepatocellular carci- noma. Hepatology 1998;27:273-278.

4. Terentiev AA, Moldogazieva NT. Alpha-fetoprotein: a renaissance. Tumour Biol 2013;34:2075-2091.

5. Bruix J, Sherman M; Practice Guidelines Committee; American Association for the Study of Liver Diseases. Management of hepatocellular carcinoma. Hepatology 2005;42:1208-1236.

6. Park JW; Korean Liver Cancer Study Group and National Cancer Center. Practice guideline for diagnosis and treatment of hepatocellular carcinoma. Korean J Hepatol 2004;10:88-98.

7. Soresi M, Magliarisi C, Campagna P, et al. Usefulness of alpha-fetoprotein in the diagnosis of hepatocellular carcinoma. Anticancer Res 2003;23:1747-1753.

8. Bruix J, Sherman M; American Association for the Study of Liver Diseases. Management of hepatocellular carcinoma: an update. Hepatology 2011;53:1020-1022.

9. Cedrone A, Covino M, Caturelli E, et al. Utility of alpha-fetoprotein (AFP) in the screening of patients with virus-related chronic liver disease: does different viral etiology influence AFP levels in HCC? A study in 350 Western patients. Hepatogastroenterology 2000;47:16541658.

10. Gambarin-Gelwan M, Wolf DC, Shapiro R, Schwartz ME, Min AD. Sensitivity of commonly available screening tests in detecting hepatocellular carcinoma in cirrhotic patients undergoing liver transplantation. Am J Gastroenterol 2000;95:1535-1538.

11. Gupta S, Bent S, Kohlwes J. Test characteristics of alpha-fetoprotein for detecting hepatocellular carcinoma in patients with hepatitis C: a systematic review and critical analysis. Ann Intern Med 2003;139:46-50.

12. European Association for Study of Liver; European Organisation for Research and Treatment of Cancer. EASL-EORTC clinical practice guidelines: management of hepatocellular carcinoma. Eur J Cancer 2012;48:599-641.

13. Chan SL, Mo F, Johnson PJ, et al. Performance of serum $\alpha$-fetoprotein levels in the diagnosis of hepatocellular carcinoma in patients with a hepatic mass. HPB (Oxford) 2014;16:366-372.

14. Sanai FM, Sobki S, Bzeizi KI, et al. Assessment of alpha-fetoprotein in the diagnosis of hepatocellular carcinoma in Middle Eastern patients. Dig Dis Sci 2010;55:3568-3575.

15. Nguyen MH, Garcia RT, Simpson PW, Wright TL, Keeffe EB. Racial differences in effectiveness of alpha-fetoprotein for diagnosis of hepatocellular carcinoma in hepati- 
tis C virus cirrhosis. Hepatology 2002;36:410-417.

16. Liu C, Xiao GQ, Yan LN, et al. Value of $\alpha$-fetoprotein in association with clinicopathological features of hepatocellular carcinoma. World J Gastroenterol 2013;19:18111819.

17. Matsushita N, Hashimoto E, Tokushige K, et al. Investigation of ornithine carbamoyltransferase as a biomarker of liver cirrhosis. Intern Med 2014;53:1249-1257.

18. McGlynn KA, London WT. The global epidemiology of hepatocellular carcinoma: present and future. Clin Liver Dis 2011;15:223-243.

19. Te HS, Jensen DM. Epidemiology of hepatitis B and C viruses: a global overview. Clin Liver Dis 2010;14:1-21.

20. Trevisani F, D'Intino PE, Morselli-Labate AM, et al. Serum alpha-fetoprotein for diagnosis of hepatocellular carcinoma in patients with chronic liver disease: influence of HBsAg and anti-HCV status. J Hepatol 2001;34:570-575.

21. Marrero JA, Feng Z, Wang Y, et al. Alpha-fetoprotein, des-gamma carboxyprothrombin, and lectin-bound alpha-fetoprotein in early hepatocellular carcinoma. Gastroenterology 2009;137:110-118.

22. Xu JB, Qi FZ, Xu G, et al. Value of alpha-fetoprotein and clinical characteristics in patients with liver neoplasm. Neoplasma 2014;61:218-224. 